\title{
Change is coming: Lead, follow, or get out of the way
}

\author{
D. George Wyse
}

Received: 2 April 2009 / Accepted: 6 April 2009 / Published online: 17 July 2009

(C) Springer Science + Business Media, LLC 2009

Keywords Editorial $\cdot$ The future $\cdot$ Arrhythmia management . Change

Change is a popular theme at this particular moment in time. The word has been used a lot lately, taking on the cloak of a mantra. Some of that usage tapped into an apparent longing for something, as yet not clearly defined or realized that played a prominent part in the results of the recent American general election. It is not entirely clear to me, however, exactly what "change" means to most of us, in that or many other contexts, or whether or not most of us have really actually thought about it. Is it a noun or a verb, as in a command? It probably at least partly encompasses a desire for things to be different than they are. But what do we want changed? Is different better? How much ability and will do we have to make change happen? It seems to me that some, maybe a lot, of change happens whether we want it or not.

What we want changed is never constant over time, is not the same for everyone, and is often in polar opposite directions for any two individuals. It has been my impression over the years that many, if not most people are not particularly comfortable with change. In fact, many seem downright resistant to it. I am pretty sure most of us only want change that we think will make life better for us. If life is already comfortable, there is little incentive to change. That's not to say one necessarily knows ahead of

D. G. Wyse $(\bowtie)$

Department of Cardiac Sciences,

Libin Cardiovascular Institute of Alberta/University of Calgary,

Room G009, Health Sciences Center, 3330 Hospital Dr. NW,

Calgary, AB, Canada T2N 1N4

e-mail: dgwyse@ucalgary.ca time what change will make life better for them. Most often there is a good deal of uncertainty about what we mean by change, how to make it happen, or perhaps, how to stop it, retard it, or steer it. Let's take the example of climate change. It is mildly amusing that many seem so upset and downright fearful about something that has been around for millions of years. Truly it seems prudent to minimize any harmful effect of humanity on the environment. However, to attribute climate change mostly to human activity and to inflict us with faith-based, religious-type fervor in an effort to resist change seems misguided. Associations are just associations. After all, climate change was happening when people weren't even here (cf. the ice ages).

My theme here is not about making change happen. For those of us who toil in science that is our raison d'être. To be the instrument of change (hopefully for the better) is sublime! My theme is more that change is inevitable, often unanticipated, and not entirely controllable. We have to learn to deal with change in those terms. How we deal with inevitable, unanticipated and uncontrollable change will determine a lot about us, collectively and as individuals. Why are we so resistant to change? Do we have an inherent and primordial desire to preserve our species that makes us want to keep things the way they are (the Darwinian argument)? During my lifetime many changes, over which I have had no control, but which are important to my daily living, have taken place. Oddly enough, that is something I don't think is going to change! One has limited ability to predict what changes are going to happen and when they are going to happen. One needs to acquire a certain amount of comfort with uncertainty and the unknown. But most importantly, one must be prepared to change when circumstances change. A wise mentor once told me, "Don't be first but worse, don't be the last". Those that are prepared for 
change and can adapt to it are the ones who are going to succeed. Those that expend an inordinate amount of time and effort resisting change will ultimately fail. That's not to deny totally that trying to control the pace of change is not a good idea in some situations. It is also useful to remember the admonishment that compromise can be "a bridge to change". Just don't let compromise become an excuse to maintain the status quo.

To illustrate my theme a little more concretely, let's just look back a short distance in time for a moment, focusing on our own area of clinical cardiac electrophysiology. When I was training in clinical cardiac electrophysiology, just a little over 30 years ago now, I did not learn how to do radiofrequency ablation, manage an ICD, or implant a CRT device. Yet at the present time these activities consume much of the time of many practicing clinical cardiac electrophysiologists. In 1976 I did not have a clue what we would end up doing as clinical cardiac electrophysiologists in 2009. I am pretty confident that in 2009 I do not have a clue what you are going to be doing in clinical cardiac electrophysiology in 2042. It will be different (cf. above), however.

What seems to be eminently sensible at this point in time will be considered passe at some point in the future. For example, in the early part of my career it seemed that we were entering an era when antiarrhythmic drugs would dominate clinical cardiac electrophysiology and arrhythmia treatment for the foreseeable future. Antiarrhythmic drug therapy guided by programmed electrical stimulation also seemed so "sensible". The logic of interrogating an arrhythmia substrate by programmed stimulation, finding an antiarrhythmic drug that would prevent arrhythmia induction, and then applying that treatment for secondary arrhythmia prevention to save lives was compelling. Many, many hours were spent in this activity, but when the hypothesis was tested it was found to be seriously wanting. Similarly, the process of quantification of ventricular ectopy after a myocardial infarction and suppressing it with antiarrhythmic drugs, although logical at the time, was likewise found to be, not only futile, but harmful. In fact, the era of antiarrhythmic drugs, if it is truly over, seems to have been perilously short. Oh, that reminds me, I forgot to tell you about acceleration of the pace of change. The treatment $d u$ jour is prevalent for a shorter and shorter period.

It is an interesting intellectual exercise to reflect on why our eminently sensible antiarrhythmic drug therapy approaches of the 1980s went awry. How did we miss the mark so widely? I think part of the answer lies in the fact that we were thinking of arrhythmia as a static "disease" rather than a consequence of "disease" with a pathophysiology that is constantly waxing and waning. The model upon which we based our thinking was faulty. It is indeed true that arrhythmia is a "stand alone" disease with a wellunderstood pathophysiology in certain instances, such as, the Wolff-Parkinson-White syndrome. However, in the case of the major arrhythmia problems we currently face, ventricular tachycardia/fibrillation (the bulk of sudden arrhythmic death) and atrial fibrillation, the arrhythmia is most often actually the consequence of another "disease", whose pathophysiology waxes and wanes in ways we don't fully understand. The fact of the matter is that we didn't (and still don't) really understand the pathophysiology of these arrhythmias well enough to define the ideal properties of an antiarrhythmic drug for each individual clinical situation. Eventually, the understanding of the Wolff Parkinson-White syndrome led to the eminently rational ablative approach now used widely for that condition. The extension of a similar understanding into a unifying rationale for ablation of atrial fibrillation is in progress but has not yet been and may never be fully achieved (cf. below).

Can we predict what will change and how it will change? I already confessed above that I don't have clue what a clinical cardiac electrophysiologist will be doing in 2042. However, that doesn't deny me the fun of thinking about it. Let's look at the ICD for a minute. The ICD approach to preventing sudden arrhythmic death is intellectually parsimonious. A full understanding of the pathophysiology of sudden arrhythmic death is not required. The current major intellectual exercise is to determine who should and should not get an ICD and to make the process as cost effective as possible. If we understood the pathophysiology sudden arrhythmic death better and could alter the arrhythmia milieu with as yet undiscovered pharmacologic or biologic agents applied through individualized medicine, or if we were better at preventing heart disease, or if we could make replacement hearts from stem cells, would the ICD disappear?

Likewise let's consider ablative therapies for atrial fibrillation. At least when we ablate the atrioventricular junction and put in a pacemaker, we understand what we are doing. Intellectually, atrioventricular ablation and a pacemaker for atria fibrillation are not much different from putting in an ICD to prevent sudden arrhythmic death. Radiofrequency ablation for "curing" atrial fibrillation, on the other hand, at this moment in time seems a little precarious, a little akin to EP-guided antiarrhythmic drug therapy in 1980. It seems sensible but will it stand up to rigorous testing? That is not to deny, however, that we have learned a lot from the efforts to craft an ablative "cure" for $\mathrm{AF}$. Indeed we have. It does seem to me, however, from what we know so far about the pathophysiology of AF that the probability of radiofrequency ablation becoming the answer for permanent treatment of the vast majority of patients with atrial fibrillation is low. In its present form, we couldn't even begin to contemplate how we would deliver 
ablative therapy to the millions of patients with AF. At the moment it is in its "rapid growth phase". I have the sense that when its true value is known it will be applied to a relatively small and select number of patients with atrial fibrillation, primarily those without significant structural heart disease. Again, if we were better at primary prevention, or if we understood the pathophysiology in an individual case, or if we applied "personalized" drug therapy with as yet undiscovered drugs or biologic agents, would we do a better job than can possibly be done with ablation and will it eventually disappear?

Well, as I said, I don't have a clue what will happen. I know it will be interesting and different. Remember it's the journey not the destination that is important. 